\title{
Polarized window for left-right symmetry and a right-handed neutrino at the Large Hadron-Electron Collider
}

\author{
Subhadeep Mondal ${ }^{1, \text { f }}$ and Santosh Kumar Rai ${ }^{1, \oplus}$ \\ ${ }^{1}$ Regional Centre for Accelerator-based Particle Physics, \\ Harish-Chandra Research Institute, Chhatnag Road, Jhusi, Allahabad 211019, India
}

\begin{abstract}
The breaking of parity, a fundamental symmetry between left and right is best understood in the framework of left-right symmetric extension of the standard model. We show that the production of a heavy right-handed neutrino at the proposed Large Hadron-Electron Collider (LHeC) could give us the most simple and direct hint of the scale of this breaking in left-right symmetric theories. This production mode gives a lepton number violating signal with $\Delta L=2$ which is very clean and has practically no standard model background. We highlight that the right-handed nature of $W_{R}$ exchange which defines the left-right symmetric theories can be confirmed by using a polarized electron beam and also enhance the production rates with relatively lower beam energy.
\end{abstract}

PACS numbers: 12.60.Cn, 14.60.St, 13.15.+g

Keywords: Left-right symmetry; Right-handed neutrino, LHeC, beam polarization.

The standard model (SM) picture of particle physics has almost already seen its great success at the Large Hadron Collider (LHC) with the discovery of the Higgs boson. Notwithstanding the success, what has been more intriguing is the lack of any clear hint of physics beyond the SM (BSM) within the LHC data yet. Of course there have been some excitement with reported excesses at the kinematic thresholds of the recently concluded run-I of the LHC. But these have hardly been conclusive of anything and a better picture will definitely emerge at the run-II of LHC. However, we are already aware that the SM picture is incomplete and one of the clearest signs of BSM has been the existence of non-zero neutrinos mass. Whether the neutrinos are Majorana or Dirac type is yet to be determined but one definitely needs to invoke physics beyond the SM to generate mass for the neutrinos. The most popular of the lot is the so called seesaw mechanism [1] where light neutrino masses result after the heavier degrees have been integrated out. The Majorana nature of the neutrino would lead to a $\Delta L=2$ lepton number violating (LNV) process with its clear imprint in processes such as the neutrinoless double beta decay $(0 \nu \beta \beta)$. The possibility of observing such a process at collider experiments such as the LHC is quite difficult and requires the seesaw scale to be quite low at around the $\mathrm{TeV}$ scale and a heavy Majorana neutrino at less than a few hundred GeV [2]. The production of the right-handed neutrinos however get enhanced at LHC with new gauge bosons in the theory [3 [5]. Left-Right (LR) models [6 11] based on the gauge group

$$
\mathcal{G}_{L R}=S U(3)_{C} \otimes S U(2)_{L} \otimes S U(2)_{R} \otimes U(1)_{B-L} .
$$

were the first which naturally led to the existence of a right-handed neutrino because of the symmetry and explain the existence of tiny neutrino mass. They also lead to a clear understanding of why parity is maximally violated in weak interactions and not introduced in an $a d$ hoc manner in the theory. Thus a knowledge of the scale of this breaking would help understand one of the fundamental questions within the SM picture. In this Letter we focus on the minimal version of the left-right symmetric theory. Although unrelated, it is quite interesting to note and worth pointing out that most of the new ideas that seem to be addressing the reported excesses at run-I of LHC [12, 13] have taken refuge in the framework of left-right symmetry $14-16]$. This study underlines the prospects of uncovering such a specific symmetry at the proposed LHeC.

LHeC will be the second $e^{-} p$ collider after HERA supposed to be built at the LHC tunnel [17, 18]. The design is planned so as to collide an electron beam with a typical energy range, $60-150 \mathrm{GeV}$ with a $7 \mathrm{TeV}$ proton beam producing a parton level center-of-mass energy close to $\sqrt{\hat{s}} \simeq 1.3 \mathrm{TeV}$. It is expected to achieve an $100 \mathrm{fb}^{-1}$ integrated luminosity per year. One particular aspect of LHeC that can be very crucial is the availability of a polarized electron beam, which we show, can help in probing the left-right symmetry in nature directly.

Left-right symmetry: The left-right (LR) symmetry model keeps the SM fermion content the same but extends the SM gauge symmetry by another $S U(2)$. The minimal model is based on the gauge group $\mathcal{G}_{L R}$. The quarks and leptons are completely LR symmetric with the following representations under $\mathcal{G}_{L R}$ :

$$
\begin{aligned}
Q_{L} & \sim(3,2,1,1 / 3), & & \ell_{L} \sim(1,2,1,-1) \\
Q_{R} & \sim(3,1,2,1 / 3), & & \ell_{R} \sim(1,1,2,-1)
\end{aligned}
$$

The formula for the electromagnetic charge $(Q)$ is given by

$$
Q=T_{3 L}+T_{3 R}+\frac{B-L}{2}
$$

where $T_{3 L / 3 R}$ are the Isospin generators of $S U(2)_{L / R}$. The symmetry breaking pattern of the LR gauge symme- 
try down to the SM gauge group and finally to $U(1)_{e m}$ is brought about by giving VEV to the the $S U(2)_{L, R}$ triplets $\Delta_{L}(3,1,2)$ and $\Delta_{R}(1,3,2)$ and a Higgs bi-doublet $\Phi(2,2,0)$ where the charges in parentheses are according to the $S U(2)_{L} \times S U(2)_{R} \times U(1)_{B-L}$ quantum numbers.

The VEV structure responsible for the above symmetry breaking is as follows:

$$
\left\langle\Delta_{L}\right\rangle=0,\left\langle\Delta_{R}\right\rangle=\left[\begin{array}{cc}
0 & 0 \\
v_{R} & 0
\end{array}\right],\langle\Phi\rangle=\left[\begin{array}{cc}
v_{1} & 0 \\
0 & v_{2} \mathrm{e}^{i \alpha}
\end{array}\right]
$$

where $v_{1,2}, v_{R}$ are real and positive with $v_{R} \gg v_{1,2}$. Note that the LR symmetry gets broken down to SM with $\left\langle\Delta_{R}\right\rangle$ while the SM symmetry breaking happens when the bi-doublet acquires a VEV $\langle\Phi\rangle$. The $W$ boson has a mass given by $M_{W}^{2}=g_{L}^{2} v^{2} \equiv g_{L}^{2}\left(v_{1}^{2}+v_{2}^{2}\right)$ where $g_{L}$ denotes the $S U(2)_{L}$ gauge coupling. The $\Delta_{L}$ develops a tiny $\operatorname{VEV}\left\langle\Delta_{L}\right\rangle \propto v^{2} / v_{R}$ which is crucial for the light neutrino masses.

The heavy right handed gauge boson masses (neglecting mixing) are given by

$$
M_{W_{R}}^{2} \simeq g^{2} v_{R}^{2}, \quad M_{Z_{R}}^{2} \simeq 2\left(g^{2}+g_{B-L}^{2}\right) v_{R}^{2}
$$

where the gauge couplings of hypercharge $\left(g_{Y}\right)$ and $g_{B-L}$ are related through $1 / g_{Y}^{2}=1 / g_{R}^{2}+1 / g_{B-L}^{2}$. Note that due to LR symmetry and Parity $(\mathrm{P})$ being a good symmetry, we have set $g_{L}=g_{R}=g$. Under this assumption the $M_{Z_{R}} \simeq 1.7 M_{W_{R}}$ and therefore the $W_{R}$ discovery would give us a clear and more importantly the first hint of the LR symmetry breaking scale. With the scale of LR breaking around a few $\mathrm{TeV}$ one can generate large Majorana masses for the right-handed neutrinos. This leads to the seesaw mechanism and generate tiny nonzero neutrino masses for the active neutrinos. For more details on the spectrum including that of right-handed neutrinos, and discussion on the limits of the LR scale, we refer the readers to Ref. 19 21. In fact for our case, in the minimal LR symmetry model, the heavy neutrinos masses are with similar hierarchy as the light ones $\left(M_{N} \propto m_{\nu}\right)$.

LR symmetry at $L H C$ : Irrespective of the theoretical constraints on the $M_{W_{R}}$ of about $2.5 \mathrm{TeV}$ 22, 23], the LHC data is set to improve upon the direct search limits of $M_{W_{R}} \gtrsim 3 \mathrm{TeV}$ [12] at $95 \%$ C.L. obtained at LHC with $\sqrt{s}=8 \mathrm{TeV}$. With improved center-of-mass energy and higher luminosity, this reach will only improve further with a possibility of discovery for a sub $5-6 \mathrm{TeV} W_{R}$ $[3,19]$. In addition to that it is also expected to exclude a large mass region for the heavy right-handed neutrino, as $W_{R} \rightarrow N_{R} \ell$ gives a dilepton + dijet channel which can be used to reconstruct the $W_{R}$ and $N_{R}$ mass using $M_{\ell \ell j j}$ and $M_{\ell j j}$ invariant mass distributions respectively. However one cannot rule out the possibility that a sequential $W^{\prime}$ with couplings to left-handed fermions only, might also lead to such a signal. A clear argument in favor of the

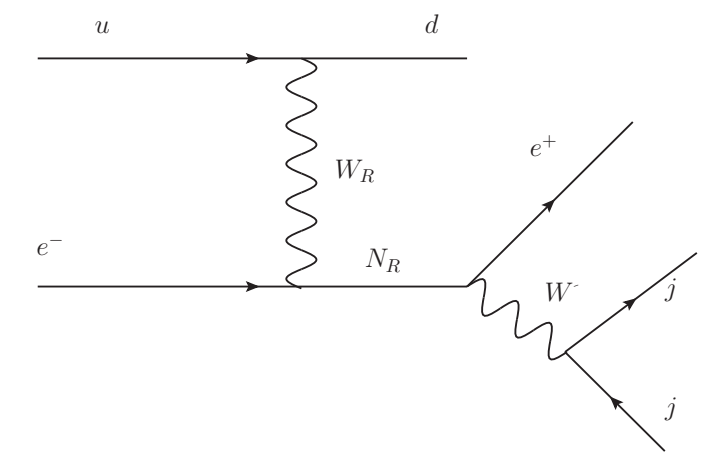

FIG. 1. Illustrating the Feynman diagram that contributes to the $N_{R} j+X$ production through the exchange of a heavy $W_{R}$ in the $t$-channel in $e^{-} p$ collision at LHeC.

LR symmetry and the signal arising from the production of a $W_{R}$ boson will be if one can verify the nature of the coupling of $W_{R}$ with the SM fermions. This is the prime objective that we wish to address through this Letter and present a study of the simplest process allowed where the LR scale is directly involved, at the proposed LHeC. To do this, we only require to highlight the charged-current interaction Lagrangian in the LR model. This has both left and right-handed charged gauge bosons with their corresponding fermionic interactions in the mass eigenstate basis:

$$
\mathcal{L}_{W}=\frac{g}{\sqrt{2}}\left(\bar{f}_{L} V_{L}^{\dagger} W_{L} f_{L}^{\prime}+\bar{f}_{R} V_{R}^{\dagger} W_{R} f_{R}^{\prime}\right)+\text { h.c. }
$$

where, when $f$ and $f^{\prime}$ stand for quarks the $V_{L / R}$ represents the CKM matrix for the left and right-handed quarks respectively, whereas for the case of $f, f^{\prime} \in$ leptons the $V_{L / R}$ represents the PMNS mixing matrix for the left-handed leptons and the corresponding analog for the right-handed leptons respectively. This interaction Lagrangian leads to the production of a heavy righthanded neutrino $N_{R}$ through the exchange of a righthanded charged gauge boson $W_{R}$ which constitutes the only dominant contribution to the process

$$
e^{-} p \rightarrow N_{R} j
$$

at LHeC, where $j \in q_{i}$ (light quarks). Thus an $e^{-} p$ collider presents us with an opportunity of testing the LR symmetry breaking scale because of the direct coupling $e-N_{R}-W_{R}$ unique to the left-right symmetric theory.

LHeC as a tool to confirm LR symmetry: As pointed out earlier, the search for a heavy right-handed neutrino $\left(N_{R}\right)$ at the LHC through the LNV $\Delta L=2$ process is quite well studied. Unlike typical $\mathrm{TeV}$-scale seesaw scenarios where the production is mediated by the SM $W$ boson the production in the LR model is mediated by the heavy $W_{R}$ gauge boson. We propose in this Letter that the $\mathrm{LHeC}$ would be an excellent place to determine such 


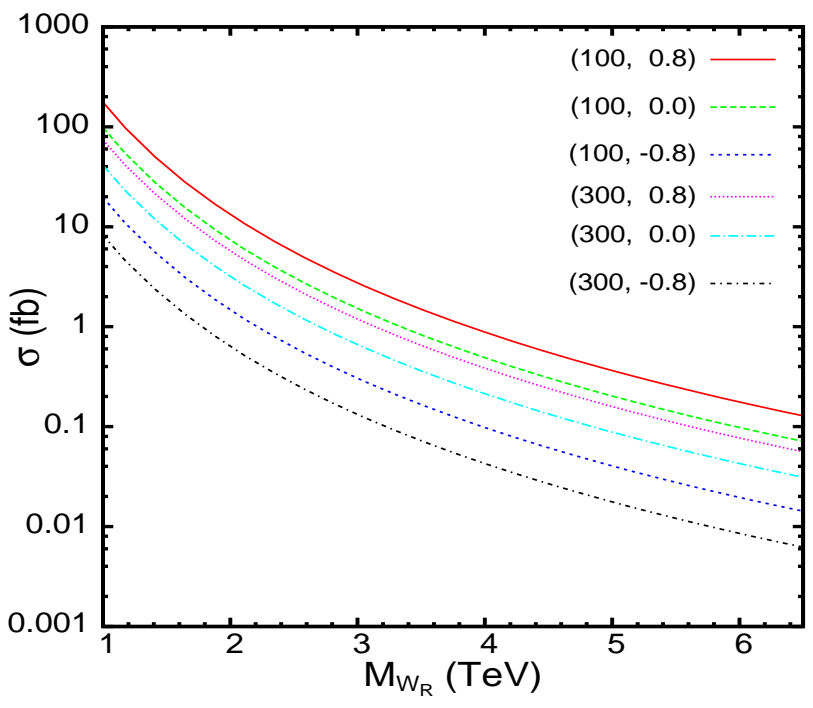

FIG. 2. The production cross section for the $N_{R} j$ at LHeC as a function of the $W_{R}$ mass. Different curves correspond to different values of $N_{R}$ mass and the choice for electron beam polarizations represented as $\left(M_{N_{R}}, P_{e}\right)$ in parentheses.

information and confirm the LR symmetry. On the downside, the LHeC loses the advantage of producing a heavy $W_{R}$ as a resonance like the LHC. Thus it might seem that it would be limited in the mass reach of $W_{R}$. However an important thing to note here is that for an $e p$ machine such as the LHeC, an exchange of the right-handed $W_{R}$ leads directly to the production of a right-handed neutrino of the electron-type, which is only possible in the LR model (see Eq. 5). This invariably makes it the minimal process possible with a $W_{R}$ exchange to probe the LR scale. In terms of the production rates for $N_{R}$, the LHeC should definitely outperform future $e^{+} e^{-}$colliders. Note that the production of a right-handed $N_{R}$ at $\mathrm{LHeC}$ also presents us with a process with negligible SM background 1 which can more than compensate for the suppressed rates compared to LHC. The process in consideration is shown in Fig 1 where we get

$$
e^{-} p \rightarrow e^{+} j+X
$$

which is the LNV $\Delta L=2$ analog of what is expected at the LHC through a Majorana neutrino production. This mode for a heavy Majorana neutrino signal has been considered before for LHeC [24 26]. We wish to highlight the fact that as the electron beam at the LHeC can be longitudinally polarized to study electroweak physics and to disentangle vector and axial vector contributions [17, this can be used to the advantage of studying the $N_{R}$

1 There may be some non-trivial SM background induced by the jet-electron misidentification. production which shall invariably also confirm any hint of a LR symmetry in nature.

We note that the LHeC will be a "ring-ring (RR)" collider with an option for the "linac-ring (LiR)" configuration too, with a linear electron accelerator tangential to the LHC. The RR option will be able to provide high luminosity but for higher electron energies in the ring, maintaining the beam polarization is difficult. Therefore the beam polarization shall decrease as the electron beam energy is ramped up. With a modest beam energy of $60 \mathrm{GeV}$ for the electron beam one can still have $80 \% \mathrm{~L} / \mathrm{R}$ beam polarization, while the proton beam carries $7 \mathrm{TeV}$ energy. The LiR configuration however has a high potential for polarised electrons. We use the RR configuration to present our results noting that improved energies at $\mathrm{LiR}$ for the electron beam while maintaining the polarization would only help to enhance the signal rates further.

Analysis and results: The $e^{-} p \rightarrow N_{R} j$ production cross section is calculated in the LR symmetry model using the package CalcHEP 27]. We have used the model files for the minimal model provided in Ref [21]. For the initial quarks we use the CTEQ6L1 parton distribution functions (PDF) 28]. The proton has $E_{p}=7 \mathrm{TeV}$ while the electron beam has $E_{e}=60 \mathrm{GeV}$. We take the maximum available electron beam polarization $\left(P_{e}\right)$ to be $80 \%$. In Fig. 2 we plot the production cross section of $e^{-} p \rightarrow N_{R} j$ as a function of the $W_{R}$ mass for two different values of the heavy neutrino mass $M_{N_{R}}=100,300$ $\mathrm{GeV}$. We consider three different initial beam configurations for the electron, i.e. when it is left-polarized $\left(P_{e}=-0.8\right)$, unpolarized $\left(P_{e}=0\right)$ and right-polarized $\left(P_{e}=0.8\right)$. While estimating the production cross section, we set some basic acceptance cuts for the massless quarks representing $j$ produced at the parton level, as $p_{T}^{j}>10 \mathrm{GeV}$ and $\left|\eta_{j}\right|<4$. As one would expect, due to the right-handed nature of interaction there is a clear enhancement in the production rates with the electron beam right-polarized, when compared to the unpolarized beam. In fact we find that the production rates for $N_{R} j$ using an unpolarized electron beam with $E_{e}=110$ $\mathrm{GeV}$ is similar to the cross section for $E_{e}=60 \mathrm{GeV}$ and $P_{e}=0.8$. In addition, the cross section would drop considerably once the beam polarization is reversed. Ideally for a $100 \%$ left polarized beam there would hardly be any contribution to the $N_{R}$ production in the LR symmetric model. However, with $80 \%$ left-polarized electron beam one is still able to get a small fraction of the $W_{R}$ contribution as shown in Fig. 2. We also find that LHeC could be sensitive to similar or even greater values of $W_{R}$ mass that could be excluded at the LHC, provided the righthanded neutrino is not too heavy. For example, when $M_{W_{R}}=6 \mathrm{TeV}$ and the electron beam is right-polarized $\left(P_{e}=0.8\right)$, we get $\sigma\left(N_{R} j\right) \sim 0.08 \mathrm{fb}$ for $M_{N_{R}}=300$ $\mathrm{GeV}$ while $\sigma\left(N_{R} j\right) \sim 0.18 \mathrm{fb}$ for $M_{N_{R}}=100 \mathrm{GeV}$. That 


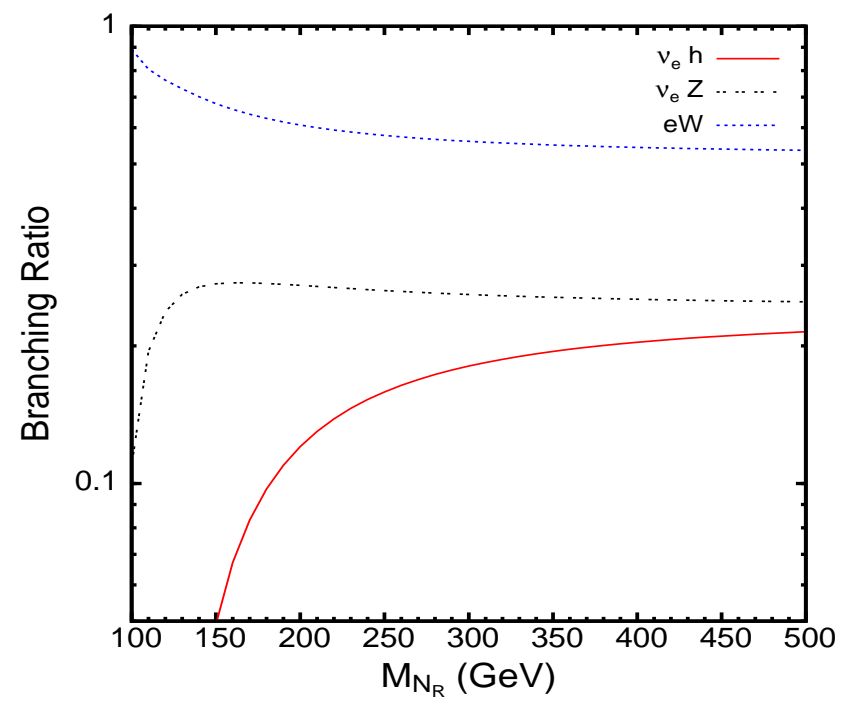

FIG. 3. Illustrating the relevant branching fractions of the right-handed neutrino $N_{R}$ decay modes as a function of its mass. Note that the $e W$ mode represents the sum of equal contributions from $N_{R} \rightarrow e^{+} W^{-}$and $N_{R} \rightarrow e^{-} W^{+}$.

corresponds to about 8 and 18 events respectively, with an $100 \mathrm{fb}^{-1}$ integrated luminosity. We consider the $e^{+} X$ final state which arises when

$$
N_{R} \rightarrow e^{+} W^{-} \rightarrow e^{+} j j,
$$

where $W$ decays hadronically with $\sim 0.7$ branching fraction. A quick look at the decay modes of the heavy neutrino, shown in Fig. 3 gives about $30 \%$ branching for $M_{N_{R}}=100 \mathrm{GeV}$ and about $21 \%$ for $M_{N_{R}}=300 \mathrm{GeV}$ to $e^{+} W^{-} \rightarrow e^{+} 2 j$ mode. This would give us nearly $5 \mathcal{A}$ events for $M_{N_{R}}=100 \mathrm{GeV}$ and $2 \mathcal{A}$ events for $M_{N_{R}}=300$ $\mathrm{GeV}$ respectively. Here we denote $\mathcal{A}$ as the acceptance efficiency for events after basic trigger cuts for the final state $e^{+} X$, which we expect would be sufficiently high while putting basic acceptance cuts for a final state with practically no SM background. The event rates are naturally greater as we go lower in the $W_{R}$ mass and we find that it nearly doubles when $M_{W_{R}}=5 \mathrm{TeV}$. Note that the change in $M_{W_{R}}$ does not affect $N_{R}$ decay branchings much. We show the event rates scaled by acceptance efficiency $(\mathcal{A})$ for the above final state in Fig. 4 for different $W_{R}$ mass. Note that as there is absolutely no $S M$ background for the above final state, an observation of even a very few distinct events would be a clear signal for the existence of a left-right symmetry in nature. In addition a clear and complementary confirmation would come through the vanishing of the same signal events once the electron beam polarization is flipped. Note that in other scenarios of heavy Majorana neutrino with no right-handed symmetry, the couplings are left-handed in nature and therefore a right-polarized beam would give no signal events while a left-polarized beam will enhance such a signal. If high enough beam polarization could be

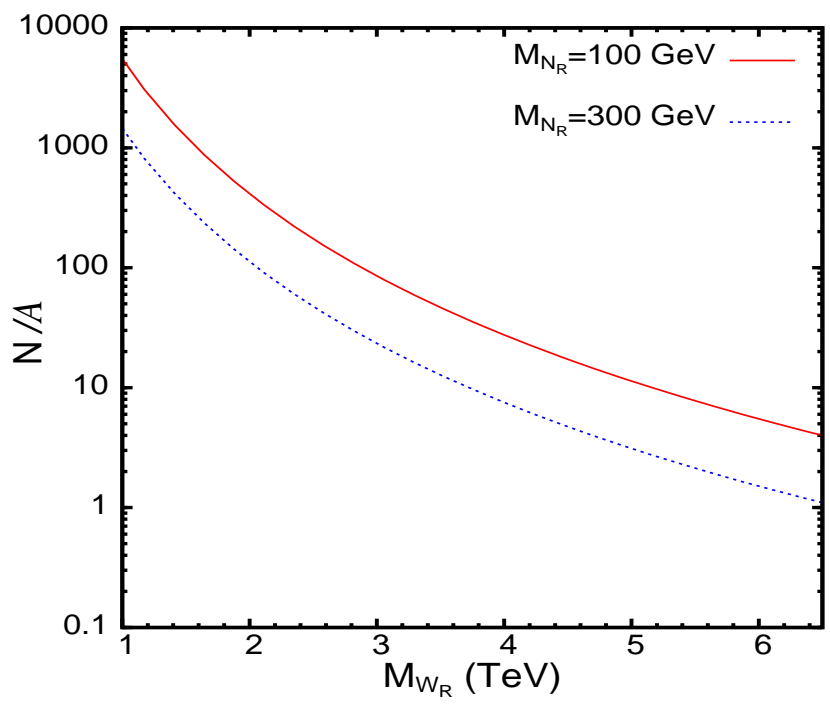

FIG. 4. The event rate at $100 \mathrm{fb}^{-1}$ luminosity normalized with acceptance efficiency $(\mathcal{A})$ at LHeC as a function of the $W_{R}$ mass with the electron beam polarization $P_{e}=0.8$.

maintained for higher electron energies too, that would clearly help in increasing the rates further.

Summary and Conclusions: To summarize, in this Letter we have shown that the proposed $e p$ collider, LHeC could be an excellent place to test the existence of LR symmetry in nature and understand why parity symmetry is maximally violated in weak interactions. LR model is the most natural extension to the SM where right-handed neutrinos appear naturally and also help us understand that parity as a symmetry is broken at a high scale. A TeV scale breaking leads to interesting collider signals. Although much lower energies compared to the LHC would be available, we show that the sensitivity can be quite high and comparable with the LHC through the production of a right-handed heavy neutrino. We also find that using a longitudinally polarized electron beam will be crucial in the search for LR symmetry at $\mathrm{LHeC}$. The correct polarization not only enhances the right-handed signal but also distinguishes it from any competing scenarios of neutrino mass generation models, which is difficult at the LHC. There could also be other possible new physics studies, using the polarized electron beam option at LHeC [29]. We must however acknowledge the fact that the LR signal might not show once the right handed neutrinos also become too heavy. In such a scenario, to maintain a polarized beam with higher energies, one would have to use the linac type setup (LiR) for the electron beam [30]. For a linac solution the electron beam polarization would be independent of beam energy and therefore a polarized electron beam with higher energies can be used at such ep colliders. Thus we find that having the electron beam polarized at LHeC not only helps in studying the electroweak physics within SM, but 
can prove to be a novel feature in unravelling one of the fundamental mysteries i.e. Parity as a symmetry in nature and the existence of left-right symmetry.

Acknowledgments: This work was partially supported by funding available from the Department of Atomic Energy, Government of India, for the Regional Centre for Accelerator-based Particle Physics (RECAPP), HarishChandra Research Institute.

* subhadeepmondal@hri.res.in

† skrai@hri.res.in

[1] P. Minkowski, Phys. Lett. B 67 (1977) 421. T. Yanagida, proceedings of the Workshop on Unified Theories and Baryon Number in the Universe, Tsukuba, 1979, eds. A. Sawada, A. Sugamoto, KEK Report No. 79-18, Tsukuba. S. Glashow, in Quarks and Leptons, Cargèse 1979, eds. M. Lévy. et al., (Plenum, 1980, New York). M. GellMann, P. Ramond, R. Slansky, proceedings of the Supergravity Stony Brook Workshop, New York, 1979, eds. P. Van Niewenhuizen, D. Freeman (North-Holland, Amsterdam). R. Mohapatra, G. Senjanović, Phys.Rev.Lett. 44 (1980) 912

[2] T. Han and B. Zhang, Phys. Rev. Lett. 97, 171804 (2006) hep-ph/0604064.

[3] A. Ferrari, J. Collot, M. L. Andrieux, B. Belhorma, P. de Saintignon, J. Y. Hostachy, P. Martin and M. Wielers, Phys. Rev. D 62, 013001 (2000).

[4] K. Huitu, S. Khalil, H. Okada and S. K. Rai, Phys. Rev. Lett. 101, 181802 (2008) arXiv:0803.2799 [hep-ph]].

[5] P. S. B. Dev, A. Pilaftsis and U. k. Yang, Phys. Rev. Lett. 112, no. 8, 081801 (2014) arXiv:1308.2209 [hep-ph]].

[6] J. C. Pati and A. Salam, Phys. Rev. D 10 (1974) 275.

[7] R. N. Mohapatra and J. C. Pati, Phys. Rev. D 11 (1975) 2558.

[8] G. Senjanović and R. N. Mohapatra, Phys. Rev. D 12 (1975) 1502.

[9] G. Senjanović, Nucl. Phys. B 153 (1979) 334.

[10] R. N. Mohapatra and G. Senjanović, Phys. Rev. Lett. 44, 912 (1980).

[11] R. N. Mohapatra and G. Senjanović, Phys. Rev. D 23, 165 (1981).

[12] V. Khachatryan et al. [CMS Collaboration], Eur. Phys.
J. C 74, no. 11, 3149 (2014) arXiv:1407.3683 [hep-ex]].

[13] G. Aad et al. [ATLAS Collaboration], arXiv:1506.00962 [hep-ex].

[14] F. F. Deppisch, T. E. Gonzalo, S. Patra, N. Sahu and U. Sarkar, Phys. Rev. D 90, no. 5, 053014 (2014) arXiv:1407.5384 [hep-ph]].

[15] J. Brehmer, J. Hewett, J. Kopp, T. Rizzo and J. Tattersall, JHEP 1510, 182 (2015) arXiv:1507.00013 [hep-ph]].

[16] P. S. Bhupal Dev and R. N. Mohapatra, Phys. Rev. Lett. 115, no. 18, 181803 (2015) arXiv:1508.02277 [hep-ph]].

[17] J. L. Abelleira Fernandez et al. [LHeC Study Group Collaboration], J. Phys. G 39, 075001 (2012) arXiv:1206.2913 [physics.acc-ph]].

[18] O. Bruening and M. Klein, Mod. Phys. Lett. A 28, no. 16, 1330011 (2013) arXiv:1305.2090 [physics.acc-ph]].

[19] A. Maiezza, M. Nemevšek, F. Nesti and G. Senjanović, Phys. Rev. D82 (2010) 055022 arXiv:1005.5160 [hep$\mathrm{ph}]$.

[20] C. Y. Chen, P. S. B. Dev and R. N. Mohapatra, Phys. Rev. D 88, 033014 (2013) arXiv:1306.2342 [hep-ph]].

[21] A. Roitgrund, G. Eilam and S. Bar-Shalom, arXiv:1401.3345 [hep-ph].

[22] Y. Zhang, H. An, X. Ji and R. N. Mohapatra, Nucl. Phys. B 802, 247 (2008) arXiv:0712.4218 [hep-ph]].

[23] G. Beall, M. Bander and A. Soni, Phys. Rev. Lett. 48, 848 (1982).

[24] H. Liang, X. G. He, W. G. Ma, S. M. Wang and R. Y. Zhang, JHEP 1009, 023 (2010) arXiv:1006.5534 [hep-ph]].

[25] C. Blaksley, M. Blennow, F. Bonnet, P. Coloma and E. Fernandez-Martinez, Nucl. Phys. B 852, 353 (2011) arXiv:1105.0308 [hep-ph]].

[26] L. Duarte, G. A. Gonzlez-Sprinberg and O. A. Sampayo, Phys. Rev. D 91, no. 5, 053007 (2015) arXiv:1412.1433 [hep-ph]].

[27] A. Belyaev, N. D. Christensen and A. Pukhov, Comput. Phys. Commun. 184, 1729 (2013) arXiv:1207.6082 [hep$\mathrm{ph}]$.

[28] J. Pumplin, D. R. Stump, J. Huston, H. L. Lai, P. M. Nadolsky and W. K. Tung, JHEP 0207, 012 (2002) hep-ph/0201195.

[29] I. T. Cakir, O. Cakir, A. Senol and A. T. Tasci, Acta Phys. Polon. B 45, no. 10, 1947 (2014) arXiv:1406.7696 [hep-ph]].

[30] U. Kaya, M. Sahin and S. Sultansoy, arXiv:1502.04115 [hep-ph]. 